\title{
How can we reduce violence and aggression in psychiatric inpatient units?
}

\author{
Arokia Antonysamy
}

\begin{abstract}
The inpatient environments in psychiatric units are not always conducive to patients' recovery. Male patients can easily feel bored especially when they are not interested in indoor activities like arts and crafts. Outdoor activities were little explored in our psychiatric intensive care unit and partly this may reflect a 'risk averse' approach. Like a pressure cooker, the patients' anger and frustration build up and unfortunately they may lash out on staff and other patients placing them at risk. The incidents of violence and aggression in our unit rose to 482 in 2011.
\end{abstract}

The Blackpool zoo was close to our unit and it was felt that our patients may benefit a weekly trip to the zoo. Other activities like computer and gym sessions were maintained. Although there were initial reluctance and anxiety amongst staff to escort patients outside the unit, regular support and encouragement made them more confident and less risk averse. Our patients provided lots of positive feedback and felt better equipped to 'fit in' with their community after discharge. The initial discrimination against our patients at the zoo slowly transformed into partnership working and the authorities at the zoo have offered a training programme for our patients on animal care and hygiene. Over a period of 12 months, the incidence of aggression and violence in the ward reduced to 126 . The average length of stay reduced by about $50 \%$. We also discharged patients who recovered remarkably well, directly from the unit rather than stepping down to acute wards. Staff motivation and enthusiasm continued to improve and this was reflected in the reduction in staff sickness rates by more than $50 \%$. Student nurses and doctors were able to understand the positive aspects of patients' lives and skills and felt able to boost their hope and determination.

\section{Problem}

Aggression and violence are common in psychiatric wards especially in psychiatric intensive care units and amongst detained patients with psychotic symptoms and personality issues. This does not mean that they are dangerous, instead adequate management may actually help them recover and reduce the need for restrictions whilst caring for them. The use of restraint and seclusion does not help in reducing the aggression levels and it may either exacerbate or maintain the aggression and violence in the unit. The use of PRN (as required) medications and secluding patients without actively engaging them in any therapeutic activity does not help in addressing patients' needs appropriately and makes them more lethargic and less able to express even normal emotions. Attending to patients' needs may not necessarily mean addressing the risks and there is a tendency to focus only on the latter due to perceptions that risk is the only problem. The activities in psychiatric intensive care units are limited and wards almost appear empty due to fear that patients may damage the pool table or break the rackets. Patients are expected to reach a reasonable level of stability in their mental state before they can be escorted to the occupational therapy room or the gym. But how can we get them to that stable phase whilst in PICU without drowsing them with medications?

\section{Background}

Lack of structured activities in psychiatric units and the resulting boredom increases the aggression and violent incidents in the ward and the assaults rise during the evenings and weekends (4). The national audit carried out by the Healthcare Commission(4) report also highlighted the impact of patient aggression on the duration of the recovery process and staff satisfaction with their jobs. The patients in psychiatric intensive care units are more likely to get abusive towards others(5) than those in acute wards. Male patients are more likely to become aggressive towards staff and other patients. They usually suffer from a diagnosis of schizophrenia $(2,3)$ or bipolar or personality disorder and lack of access to outside space further contributes to their hostile and inappropriate behaviour. Mentally ill patients have expressed the need for staff to spend more face to face sessions with them and not behind their desks doing paper work(1). The high volumes of paper work have prevented staff from spending more time with their patients which is what they are trained for(4).

\section{Baseline measurement}

The Trust strategy on management of violence and aggression (2008, HSO 15) in line with NICE (2005) recommends that service users identified to be at risk of disturbed/violent behaviour should be given the opportunity to have their needs and wishes recorded in the form of an advance directive or statement. Our Trust was the 3rd highest in reports of violence and aggression in the ward (Advancing Quality Alliance report, 2011), however it should be noted that even the most trivial episode of agression be it a verbal abuse or an aggressive gesture were captured in the data. The rates of AWOLs (Absconded without leave) were also high. This affected staff morale in the wards with consecutive increase in staff sickness. Our PICU (Psychiatric intensive care unit) was one of the worst affected area. There were high rates of aggression and violence and the unit accounted for 1/4th of the Trust's rates for 
aggression in 2011. This also affected staff's coping levels with the stress of the job with increasing sickness rates resulting in 247 reports of sickness in the unit in about six months in 2011.

The data on aggression and violence rates were obtained from the Trust audit department. Other data including AWOL, average length of stay was provided by the performance team. Staff sickness and seclusion data were obtained from the ward records.

\section{Design}

We were keen on using the skills and resources available in the ward to improve safety measures and we also felt that this initiative will empower our staff and make them feel valued and supported. All the staffs in our ward were part of this project. A team away day was organised to discuss the issues in the ward and to obtain ideas from staff that can inform our initiatives. The current position in relation to violence and aggression rates, staff sickness and poor feedback from external visits were discussed. Despite these challenges, we noticed some strengths like enthusiastic and motivated staff who were willing to change the status quo. Discussions around advanced directives with the patients highlighted their increased boredom and limited interests in traditional therapeutic activities in the ward and occupational therapy activities like arts and crafts. Our objective was to implement an innovative initiative aimed at addressing patient boredom that was identified as a major contributor to violence and aggression. The Blackpool Zoo was near to the unit and we considered providing the opportunity for our patients to visit the zoo that will also allow the OT to perform an 'in vivo' assessment of their skills including risk handling, communication with staff and public, budgeting, interaction with animals, social skills, behaviour in public and handling stresses like waiting in queues and working in teams.

This initiative did not involve any investment and we didnt have to go through the bureaucratic processes. As this project idea was well received by staff and patients alike, we felt we could implement it at a faster pace.

\section{Strategy}

The vision for the unit to become the unit of excellence was shared amongst all the staff in the PICU. The aim of the project to facilitate a patient centred recovery was made clear to the multidisciplinary team including consultant, ward manager, team leader, occupational therapist, ward nurses, junior doctor and support workers. The primary outcome measures included reduction in the incidents of violence and aggression. The secondary outcome measures included reduction in average length of stay, reduction in seclusion rates, direct discharges to community from PICU for a selected group of patients who may not cope well in stimulating environments like the acute wards, reduction in staff sickness rates with a consequent reduction in use of bank and agency staff. These outcomes were established with staff and progress made was discussed with staff at ward governance meetings held monthly.

A daily morning meeting is held involving two or more staff to provide a patient update. Those patients who can be tried on leave outside hospital grounds are identified and sent on escorted leave with staff on walking trips. When this is successful, the patients are booked for the zoo visits with staff nurses and occupational therapist. Initially the zoo trips were tried without the walking trips and this did not help all the patients because some of them felt too anxious and could not cope in that 'real' environment for long. Walking trips to the nearby park helped them to warm up to the idea of getting used to the busier situation at the zoo. The outdoor trips are used to build on the therapeutic relationship. Whilst nurses make key observations around patients' behaviour, mood, thoughts and perceptions in the external environment, the occupational therapist assess their independent functioning skills including budget handling, risk awareness, social skills and communication skills. Weekly feedback is provided about the leave outcomes for each patient.

At the end of three months, we began to hear positive feedback from patients. This included quotes like, 'thank you for trusting us and taking us out', 'I thought l'll be locked up here forever, but it is a totally different experience here and I will miss you all', 'when I was out, I thought of absconding, but you all trusted me so much that I just couldn't do it'. Carers also provided positive feedback to us and some of them wrote to the chief executive appreciating staff commitment to this difficult client group. The CQC (Care Quality Commission) visited the unit and gave a very positive feedback and said that the patient care plans were one of the best they have ever seen, as it was very much tailored to patients' needs. The confidence of the team increased to the degree that the PICU began to treat even very disturbed dementia patients with complex behavioural symptoms very skilfully.

Staff were able to share their skills and learning with medical and nursing students. These students accompanied patients to the zoo and were able to see the hidden strengths of these patients which they wouldn't have seen, had patients been confined to the unit without access to outdoor activities. The AQuA (Advancing Quality Alliance) group visited our ward to learn about the improvements made and strategies used to improve the quality of care to patients in terms of patient safety and patient experience. The improvements were sustained even after a year.

\section{Results}

Our innovative project led to a significant reduction in violence and aggression as shown in Figure 2. Along with the zoo visit, we also incorporated other initiatives like breakfast club for patients, walking trips and training at the gym. The average length of stay for our patients reduced from 90 to 30 days for an initial period and then remained stable at 55 days (Fig 3 ). The seclusion rates reduced significantly as also our staff sickness rates (Figure 3). Patients provided positive feedback about the zoo visit and apart from one delayed return to the ward, there were no reports of AWOL. Our team were more geared to calculated positive risk taking and we successfully discharged more than 25 patients directly to the community in 2012, out of which only two were readmitted in four to six weeks due to noncompliance. 
See supplementary file: ds1770.docx

\section{Lessons and limitations}

Lessons learnt:

1. We shared our learning with other teams only at a later stage. We could have collaborated with them at an earlier stage as this would have benefited acute ward patients and PICU patients alike. We realised this after one of the patients smashed the furniture in his room and transferred from the acute ward to our PICU unit. This patient stated that he heard positive stories ('less restrictions and timely discharge') about our PICU and wanted to be transferred here.

2. The staffs at the zoo were willing to deliver training sessions for our patients in animal care and hygiene with certificates appropriate to the training stage. We felt we could have explored other opportunities in other areas providing a range of choices for our patients.

3. We relied more on particular members of staff as they were the champions of the project. It was only late we realised that we should have encouraged those staff to train the others in the unit to become champions like them. This training is currently in progress

Limitations:

1. This project included only the PICU and therefore the sample size is small, although the volume of activity can be very high.

2. PICUs are better resourced than acute wards and therefore this initiative may not be successful if replicated in acute wards without adjustments to staffing levels. However patients are likely to be more settled in acute wards and may not need similar resources for escorted leave as PICUs.

Cost implications: This project was carried out at no extra cost utilising the resources available in the PICU and developing partnership working with the local council staff at the zoo. The aim to improve quality through recovery focused care actually led to efficiencies in relation to bed occupancy from about 90 days average per patient to 55 days. The PICU bed per day per patient costs about $£ 600$ and a saving of 30 days per patient relates to an approximate $£ 18000$.

\section{Conclusion}

We were able to achieve the aims of our project in delivering recovery focused care for our patients. The use of resources outside the hospital helped us to seek collaboration with partner organisations like the local authority and third sector. These partnerships helped to challenge the public's perception of mental illness. Their acceptance of our patients helped this vulnerable group to re-integrate confidently into their own communities. Although tacking stigma was not one of our key intentions, we were very pleased to note that our patients felt less stigmatised and they found the staff at the zoo very friendly and approachable. Our unit has been identified for best practice and similar initiatives are undertaken in other wards. We are keen to expand our partnership with other agencies seeking all opportunities we can to support our patients and helping them achieve their full potential and building on their hopes and aspirations.

\section{References}

1. Antonysamy A, Wieck A, Wittkowski A. Service satisfaction on discharge from a psychiatric mother and baby unit: a representative patient survey. Arch Womens Ment Health 2009;(12):359âDL362

2. Barlow K, Grenyer B, Ilkiw-Lavalle O.Prevalence and precipitants of aggression in psychiatric inpatient units. Australian and New Zealand Journal of Psychiatry 2000; (34): 967-974

3. Bowers L, Stewart D, Papadopoulos C, Dack C, Ross J, Khanom $H$, Jeffrey D (2011) Inpatient violence and aggression: A literature review

4. Healthcare Commission, National Audit of Violence (2003-2005).

5. Loubser I, Chaplin R, Quirk A. Violence, alcohol and drugs: The views of nurses and patients on psychiatric intensive care units, acute adult wards and forensic wards. Journal of Psychiatric Intensive Care. 2009; (5):33-39

\section{Declaration of interests}

None

\section{Acknowledgements}

Ms Rachel Brown, Dr Shekar Gangaraju, Ms Shirley McCrorie, Ms Sandra Ames 